\title{
Histidine 90 Function in 4-Chlorobenzoyl-Coenzyme A Dehalogenase Catalysis ${ }^{\dagger, \ddagger}$
}

\author{
Wenhai Zhang, Yansheng Wei, Lusong Luo, Kimberly L. Taylor, ${ }^{\S}$ Guang Yang," and Debra Dunaway-Mariano* \\ Department of Chemistry, University of New Mexico, Albuquerque, New Mexico 87131 \\ Matthew M. Benning ${ }^{\perp}$ and Hazel M. Holden* \\ Department of Biochemistry, 433 Babcock Drive, University of Wisconsin, Madison, Wisconsin 53706-1544
}

Received July 10, 2001; Revised Manuscript Received September 12, 2001

\begin{abstract}
Chlorobenzoyl-coenzyme A (4-CBA-CoA) dehalogenase catalyzes the hydrolytic dehalogenation of 4-CBA-CoA by attack of Asp145 on the $\mathrm{C}(4)$ of the substrate benzoyl ring to form a Meisenheimer intermediate (EMc), followed by expulsion of chloride ion to form an arylated enzyme intermediate (EAr) and, finally, ester hydrolysis in EAr to form 4-hydroxybenzoyl-CoA (4-HBA-CoA). This study examines the contribution of the active site His 90 to catalysis of this reaction pathway. The His90 residue was replaced with glutamine by site-directed mutagenesis. X-ray crystallographic analysis of H90Q dehalogenase complexed with 4-HBA-CoA revealed that the positions of the catalytic groups are unchanged from those observed in the structure of the 4-HBA-CoA-wild-type dehalogenase complex. The one exception is the Gln90 side chain, which is rotated away from the position of the His90 side chain. The vacated His90 site is occupied by two water molecules. Kinetic techniques were used to evaluate ligand binding and catalytic turnover rates in the wild-type and H90Q mutant dehalogenases. The rate constants for 4-CBA-CoA (both $7 \mu \mathrm{M}^{-1} \mathrm{~s}^{-1}$ ) and 4-HBA-CoA (33 and $11 \mu \mathrm{M}^{-1} \mathrm{~s}^{-1}$ ) binding to the two dehalogenases are similar in value. For wild-type dehalogenase, the rate constant for a single turnover is $2.3 \mathrm{~s}^{-1}$ while that for multiple turnovers is $0.7 \mathrm{~s}^{-1}$. For H90Q dehalogenase, these rate constants are 1.6 $\times 10^{-2}$ and $2 \times 10^{-4} \mathrm{~s}^{-1}$. The rate constants for EMc formation in wild-type and mutant dehalogenase are $\sim 200 \mathrm{~s}^{-1}$ while the rate constants for EAr formation are 40 and $0.3 \mathrm{~s}^{-1}$, respectively. The rate constant for hydrolysis of EAr in wild-type dehalogenase is $20 \mathrm{~s}^{-1}$ and in the H90Q mutant, $0.13 \mathrm{~s}^{-1}$. The 133fold reduction in the rate of EAr formation in the mutant may be the result of active site hydration, while the 154-fold reduction in the rate EAr hydrolysis may be the result of lost general base catalysis. Substitution of the His 90 with Gln also introduces a rate-limiting step which follows catalysis, and may involve renewing the catalytic site through a slow conformational change.
\end{abstract}

In this study, we have examined the role of the 4-chlorobenzoyl-coenzyme A (4-CBA-CoA $)^{1}$ dehalogenase active site His90 residue in catalysis of 4-CBA-CoA dehalogenation. 4-CBA-CoA dehalogenase mediates the central reaction of the 4-chlorobenzoate (4-CBA) to 4-hydroxybenzoate (4HBA) pathway found in certain soil-dwelling bacteria (Scheme 1) [reviewed in $(1,2)]$. The 4-CBA pathway provides entry into the central oxidative aromatic pathways (3) and, thus, a way for the host bacterium to utilize 4-CBA as an alternate energy source.

\footnotetext{
† This research was supported by NIH Grant GM28688 to D.D.-M. and by NIH Grant GM55513 to H.M.H.

$\ddagger$ The X-ray coordinates have been deposited as PDB entry $1 \mathrm{JXZ}$.

* To whom correspondence should be addressed. H.M.H.: Phone 608-262-4988, Fax 608-265-2904, E-mail hazel_holden@ biochem.wis.edu. D.D.-M.: Phone 505-277-3383, Fax 505-277-6202, E-maildd39@unm.edu.

§ Present address: NABI, 12276 Wilkins Ave., Rockville, MD 20852.

"Present address: SmithKline Beecham Pharmaceuticals R\&D Division, Collegeville, PA 19426-0898.

${ }^{\perp}$ Present address: BRUKER AXS, INC., 5465 E. Cheryl Parkway, Madison, WI 53711-5373.

${ }^{1}$ Abbreviations: 4-CBA, 4-chlorobenzoate; 4-CBA-CoA, 4-chlorobenzoyl-coenzyme A; 4-HBA-CoA, 4-hydroxybenzoyl-coenzyme A; Hepes, $N$-(2-hydroxyethyl)piperazine- $N^{\prime}$-2-ethanesulfonic acid; HPLC, high-performance liquid chromatography.
}

Scheme 1: The Three-Step 4-CBA Degradation Pathway Found in Certain Soil-Dwelling Bacteria
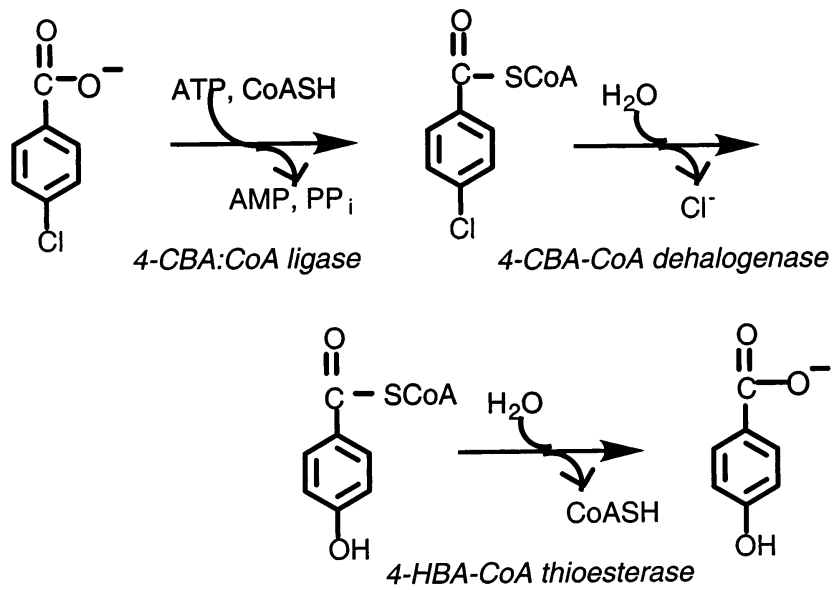

The findings derived from a series of structural, spectral, and kinetic studies of 4-CBA-CoA dehalogenase support the reaction mechanism illustrated in Figure 1A (4-14). Accordingly, binding to the active site is followed by attack of the Asp145 carboxylate group at the substrate benzoyl C(4). The Meisenheimer complex formed (henceforth referred to 

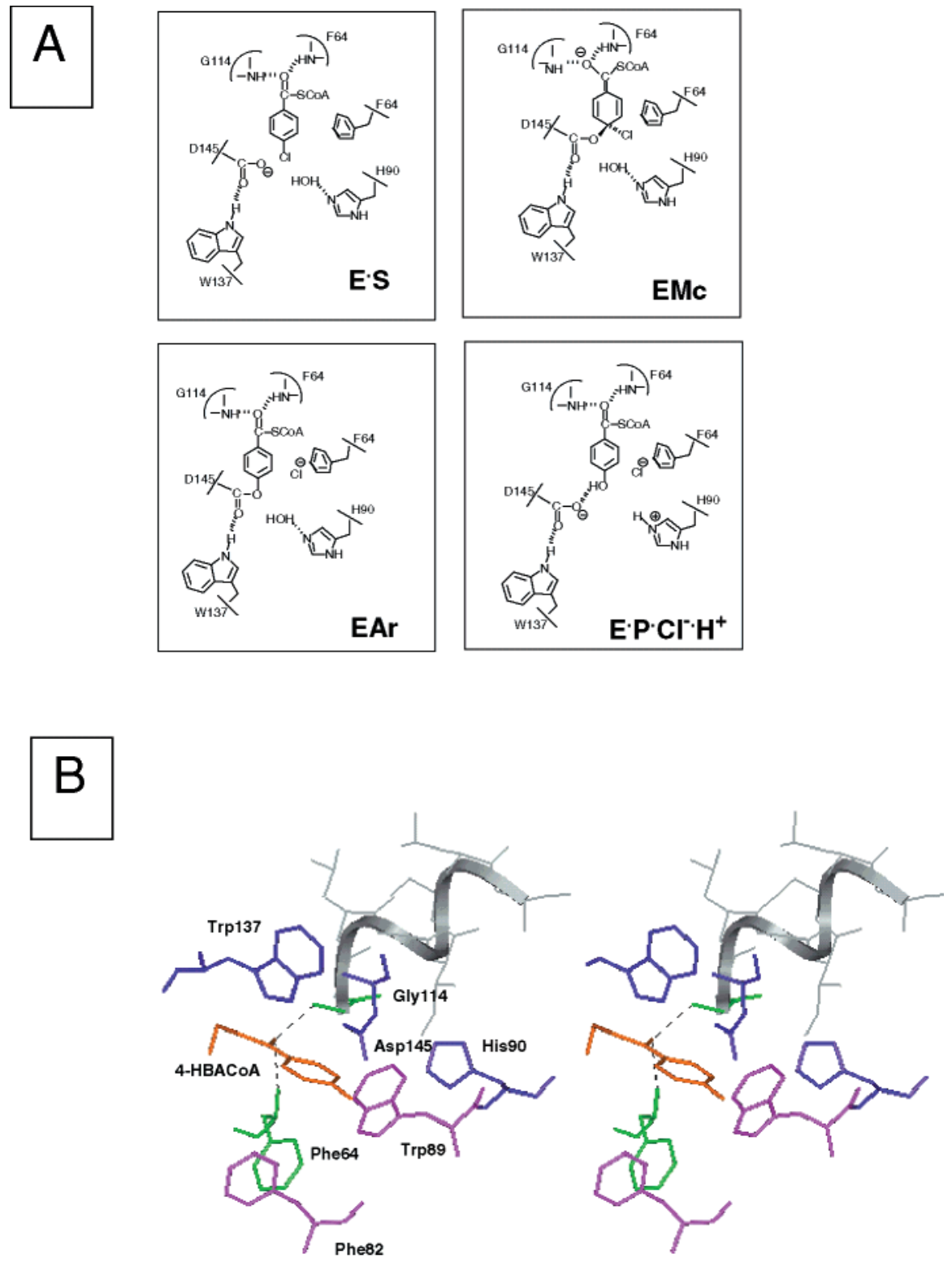

FIGURE 1: (A) Model of 4-CBA-CoA dehalogenase catalysis, picturing the catalytic residues functioning in the enzyme-substrate complex $(\mathrm{E} \cdot \mathrm{S})$, Meisenheimer intermediate $(\mathrm{EMc})$, arylated enzyme intermediate $\left(\mathrm{E} \cdot \mathrm{Ar}^{\cdot} \mathrm{Cl}^{-}\right)$, and enzyme - product $\operatorname{complex}\left(\mathrm{E} \cdot \mathrm{P} \cdot \mathrm{Cl}{ }^{-} \cdot \mathrm{H}^{+}\right) .(\mathrm{B})$ Stereodiagram of the active site region of 4-CBA-CoA dehalogenase generated from coordinates of the X-ray crystal structure of the 4-CBA-CoA dehalogenase-4-HBA-CoA complex (10) using Insight II. The polarizing H-bonds formed between the Gly114 and Phe64 backbone amide $\mathrm{NHs}$ with the benzoyl $\mathrm{C}=\mathrm{O}$ are shown with black lines.

as the EMc intermediate) expels chloride ion, to form an arylated enzyme complex (the EAr intermediate). Hydrolysis of the benzoyl ester in EAr occurs to generate the 4-HBACoA product which, along with a proton and the chloride ion, is released from the enzyme active site.

The X-ray structure of the active site region of 4-CBACoA dehalogenase with bound 4-HBA-CoA (10) (Figure 1B) reveals that the imidazole nitrogen of His 90 is positioned $3.6 \AA$ from the carbonyl carbon of the Asp145 nucleophile. From this structure, we have inferred that His90 binds and activates a water molecule for attack at the Asp145-benzoyl linkage of the EAr intermediate. To test this proposal, sitedirected mutagenesis was combined with transient kinetic analysis to measure the contribution of the His 90 side chain to catalysis. The X-ray crystal structure of the H90Q dehalogenase complex with 4-HBA-CoA was determined to provide a picture of the catalytic site within the mutant enzyme. Together, the structural and kinetic data show that
His90 functions as a barrier to solvent, as a general base in EAr hydrolysis, and as an essential component in recycling the catalytic site.

\section{EXPERIMENTAL PROCEDURES}

General. 4-CBA-CoA and 4-HBA-CoA were prepared according to (5). 4-CBA-CoA dehalogenase (specific activity $=1.5$ units $/ \mathrm{mg}$ ) was prepared according to (14) as modified in (5). H90Q dehalogenase was prepared as described in (13). $\left[{ }^{14} \mathrm{C}\right] 4-\mathrm{CBA}-\mathrm{CoA}$ (uniformly labeled on the aromatic ring) was prepared from commercial $\left[{ }^{14} \mathrm{C}\right] 4-\mathrm{CBA}$ as described in (7). The enzyme concentrations were determined by using the Bradford method (15). Enzyme spectrophotometric assays were carried out as previously described $(5,14)$. Scintillation counting was carried out with a BECKMAN LS6500 multipurpose scintillation counter. HPLC separations were carried out with a RAININ HPLC instrument equipped with a BECKMAN Ultrasphere $4.6 \mathrm{~mm} \times 25 \mathrm{~cm}$ ODS reversed- 
phase column monitored at $260 \mathrm{~nm}$ and eluted $(1.0 \mathrm{~mL} / \mathrm{min})$ with an acetonitrile/ammonium acetate gradient.

Single Turnover Reactions Measured Spectrophotometrically. Reactions were initiated by mixing $30 \mu \mathrm{L}$ of $60 \mu \mathrm{M}$ wild-type dehalogenase and $32 \mu \mathrm{L}$ of $3000 \mu \mathrm{M}$ 4-CBA-CoA or $30 \mu \mathrm{L}$ of $40 \mu \mathrm{M}$ H90Q mutant and $32 \mu \mathrm{L}$ of $400 \mu \mathrm{M}$ 4-CBA-CoA (wild-type by rapid quench and H90Q by hand). After a specified period of time, reaction solutions were quenched with $182 \mu \mathrm{L}$ of $0.2 \mathrm{M} \mathrm{HCl}$. The enzyme was separated from the quenched solution using a NANOSEP $3 \mathrm{~K}$ MW cutoff centrifugal concentrator. The $\mathrm{pH}$ of the filtrate was adjusted to 14 with $0.5 \mathrm{M} \mathrm{KOH}$. The absorbance of this solution was measured at $330 \mathrm{~nm}$ in order to determine the concentration of the 4-HBA-CoA phenolate present $(\epsilon$ $\left.=18.2 \mathrm{mM}^{-1} \mathrm{~cm}^{-1}\right)$. A plot of [4-HBA-CoA] vs time was made to show the time course for product formation during the first several turnovers. The rate constant for the first turnover catalyzed by the wild-type enzyme was calculated from the half-life $\left(t_{1 / 2}\right)$ using the equation: $k=0.693 / t_{1 / 2}$. The rate constant for ensuing turnovers was obtained from the slope of the plot following the first turnover divided by the enzyme concentration. The rate data for $\mathrm{H} 90 \mathrm{Q}$ dehalogenase were fitted using eq 1 (16). In this manner, the rate constant for the first turnover $\left(k_{1}\right)$ and the rate constant for ensuing turnovers $\left(k_{\text {cat }}\right)$ were determined

$$
\begin{gathered}
\mathrm{E} \cdot \mathrm{S} \stackrel{k_{1}}{\rightarrow} \mathrm{E} \cdot \mathrm{P} \stackrel{k_{2}}{\longrightarrow} \mathrm{E}+\mathrm{P} \\
{[\mathrm{P}]_{\mathrm{obs}}=E_{0}\{[\mathrm{EP}]+[\mathrm{P}]\}=E_{0}\left\{A_{0}\left(1-\mathrm{e}^{-\lambda t}\right)+k_{\mathrm{cat}} t\right\}}
\end{gathered}
$$

where the rates and amplitudes are defined by

$$
\begin{gathered}
\lambda=k_{1}+k_{2} \\
A_{0}=\left[k_{1} /\left(k_{1}+k_{2}\right)\right]^{2} \\
k_{\text {cat }}=k_{1} k_{2} /\left(k_{1}+k_{2}\right)
\end{gathered}
$$

$E_{0}$ is the concentration of the enzyme.

Rapid-Quench Studies. Rapid-quench experiments were carried out at $25{ }^{\circ} \mathrm{C}$ using a rapid-quench instrument from KinTek Instruments. The reactions were initiated by mixing $30 \mu \mathrm{L}$ of $156 \mu \mathrm{M}$ wild-type or H90Q dehalogenase with 32 $\mu \mathrm{L}$ of $86 \mu \mathrm{M}\left[{ }^{14} \mathrm{C}\right] 4-\mathrm{CBA}-\mathrm{CoA}$ in $50 \mathrm{mM} \mathrm{K}{ }^{+}$Hepes $(\mathrm{pH}$ 7.5) and then quenched after a specific period of time with $182 \mu \mathrm{L}$ of $0.2 \mathrm{M} \mathrm{HCl}$. The enzyme and ligand were separated from the quenched solution using a NANOSEP 3K MW cutoff centrifugal concentrator (Pall Filtron Corp.). $\left[{ }^{14} \mathrm{C}\right] 4-$ CBA-CoA and $\left[{ }^{14} \mathrm{C}\right] 4-\mathrm{HBA}-\mathrm{CoA}$ were separated from the filtrate by HPLC as described previously (7) and quantitated by liquid scintillation counting. The concentrate was subjected to 12 cycles of dilution $(500 \mu \mathrm{L}$ of $0.2 \mathrm{M} \mathrm{HCl})$ and concentration before scintillation counting. The background levels of "noncovalently bound" radiolabel present in the enzyme fractions derived from the acid-quenched reactions were accessed by using two different control reactions. For the first control reaction, the acid was added to the enzyme prior to adding the radiolabeled substrate, and for the second control reaction, the catalytically inactive D145A dehalogenase mutant was used in place of the wild-type enzyme. The amount of radioactivity observed in the enzyme fraction derived from the control reactions was routinely ca. $2-3 \%$
Scheme 2: Microscopic Rate Constants Defined for a Single Turnover Reaction Catalyzed by 4-CBA-CoA Dehalogenase

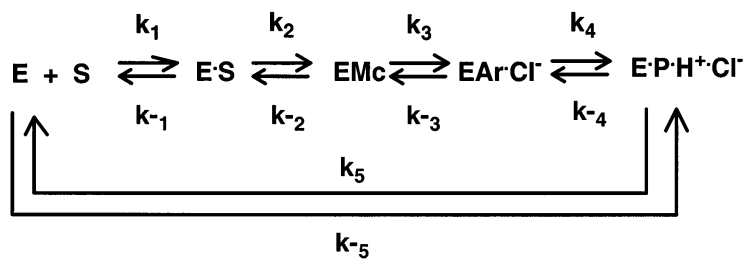

of the total radioactivity used in the reaction. This background level was subtracted from the level of radioactivity observed in the enzyme fraction generated by the test reaction.

Ligand Binding. A DX.17 MV stopped-flow spectrometer (Applied Photophysics, Leatherhead, U.K.) equipped with a $150 \mathrm{~W}$ xenon lamp was used to monitor changes in fluorescence emission (295 nm excitation, emission was monitored at $340 \mathrm{~nm}$ ) associated with the binding of 4-CBACoA or 4-HBA-CoA to wild-type or H90Q dehalogenase. The fluorescence quenching plots were analyzed using a single-exponential equation to obtain $k_{\mathrm{obs}}$. For ligand $k_{\text {on }}$ and $k_{\text {off }}$ determination, $k_{\text {obs }}$ was measured as a function of ligand concentration $(2.5-15 \mu \mathrm{M})$ at fixed enzyme concentration

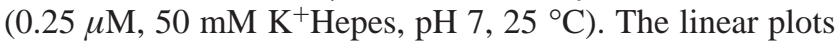
of $k_{\mathrm{obs}}$ vs the ligand were fitted to eq 2 to obtain the $k_{\text {on }}$ and $k_{\text {off. }}$

$$
k_{\mathrm{obs}}=k_{\mathrm{off}}+k_{\mathrm{on}}[\mathrm{L}]
$$

In eq $2,[\mathrm{~L}]=$ ligand concentration, $k_{\mathrm{obs}}=$ observed rate constant, $k_{\mathrm{on}}=$ the association rate constant, and $k_{\mathrm{off}}=$ the dissociation rate constant.

Kinetic Data Analysis. The progress curves for the consumption of substrate, the formation of product, and the formation-decay of radiolabeled enzyme were fitted to the kinetic model shown in Scheme 2 using the program Dynafit (17). In an independent, "blind" analysis designed to evaluate the results of the Dynafit analysis, the computer program KINSIM was used to simulate curves to the rate data using the microscopic rate constants as input (18). For wild-type dehalogenase, the microscopic rate constants were used along with eqs 3 and 4 , to calculate the steady-state $k_{\text {cat }}$ and $K_{\mathrm{m}}$. Equations 3 and 4 were derived for the kinetic model shown in Scheme 2, using the King-Altman procedure as described in (19).

$$
\begin{gathered}
k_{\text {cat }}=k_{1} k_{2} k_{3} k_{4} k_{5} / D \\
K_{\mathrm{m}}=k_{-1} k_{-2} k_{-3} k_{-4}+k_{2} k_{3} k_{4} k_{5}+k_{-2} k_{-1} k_{4} k_{5}+ \\
k_{-1} k_{-2} k_{-3} k_{5}+k_{-1} k_{5} k_{4} k_{3} / D \\
D=k_{-1} k_{-2} k_{-3} k_{-4}+k_{1} k_{4} k_{5} k_{-2}+k_{-2} k_{-3} k_{5} k_{1}+k_{1} k_{3} k_{4} k_{5}+ \\
k_{-3} k_{-4} k_{1} k_{2}+k_{1} k_{2} k_{4} k_{5}+k_{-3} k_{1} k_{2} k_{5}+k_{1} k_{2} k_{3} k_{-4}+ \\
k_{1} k_{2} k_{3} k_{5}+k_{1} k_{2} k_{3} k_{4}
\end{gathered}
$$

X-ray Crystallographic Analysis of H90Q 4-CBA-CoA Dehalogenase Complexed with 4-HBA-CoA. The details of the methodology used in the crystallization and structure determination of this complex are reported in (10). Briefly, crystals of the H90Q mutant-4-HBA-CoA complex grown from $8 \%$ poly(ethylene glycol) $8000,200 \mathrm{mM}$ potassium 


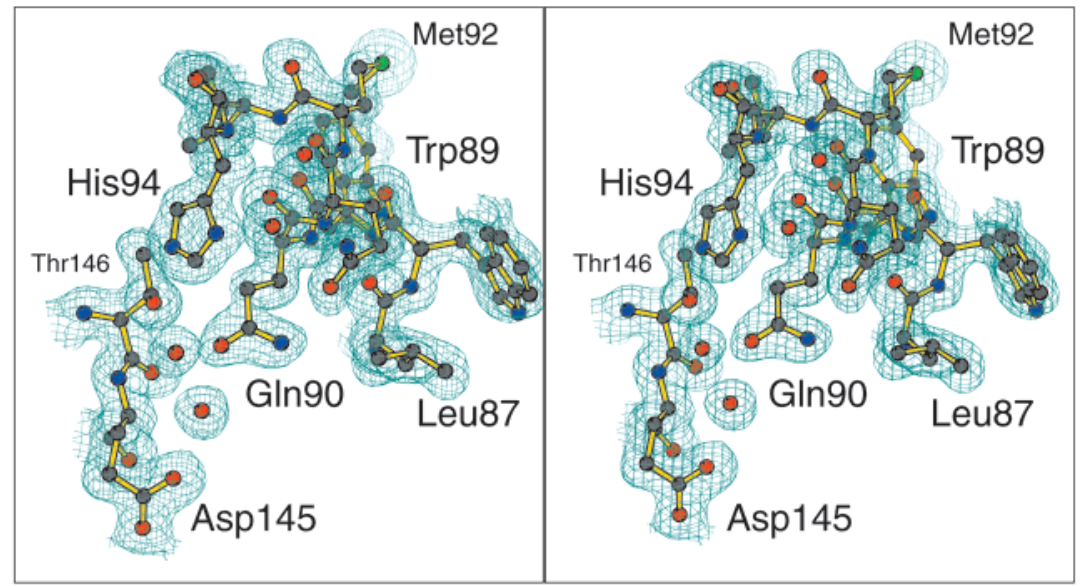

FIGURE 2: Electron density map showing the mutation site of the H90Q 4-CBA-CoA dehalogenase mutant.

chloride, $50 \mathrm{mM}$ Ches (pH 9), and $5 \mathrm{mM} \mathrm{NaN}_{3}$ at $4{ }^{\circ} \mathrm{C}$ belonged to the space group $P 2_{1} 2_{1} 2$ with unit cell dimensions of $a=107.8 \AA, b=102.4 \AA$, and $c=90.3 \AA$. Three subunits per asymmetric unit were found. The solvent content of the crystals was approximately 54\%. The X-ray diffraction maxima were observed to at least $1.8 \AA$ resolution. All X-ray data for the H90Q mutant protein and heavy atom derivative crystals [prepared using trimethyllead acetate, potassium tetrachloroplatinate(II), $p$-(hydroxymercuri)benzoate, or hexabromoridate(IV)] were collected at $4{ }^{\circ} \mathrm{C}$ with a Siemens X1000 area detector system. All X-ray data from the H90Q mutant protein and the heavy atom derivatives were processed with the data reduction software package XDS (20, 21 ) and internally scaled according to the procedure developed by Dr. Gary Wesenberg. Relevant X-ray data collection statistics are given in (10). For the H90Q protein, the overall intensity statistics are $98 \%$ completeness of data and $R$ sym $=2 \%$.

\section{RESULTS}

$X$-ray Crystal Structure of the H90Q Dehalogenase-4HBA-CoA Complex. The $\mathrm{C} \alpha$ atomic coordinates of the $\mathrm{H} 90 \mathrm{Q}$ mutant and wild-type dehalogenase molecules superimpose with an RMS deviation on $\mathrm{C} \alpha$ atoms of $0.12 \AA$. The native backbone fold is retained in the H90Q dehalogenase mutant. The electron density map at the mutation site is consistent with the replacement of His90 by a Gln residue (Figure 2).

Superpositions of the pertinent active site regions of the H90Q and wild-type dehalogenase are shown in Figure 3A,B. In the wild-type dehalogenase structure, the His 90 side chain is directed into the active site where it resides within $3.6 \AA$ of the Asp145 carboxylate. In the H90Q dehalogenase mutant, the Gln90 side chain projects away from the active site, and toward the solvent. The space occupied by the His 90 imidazole group in the wild-type enzyme is filled by two water molecules in the H90Q mutant. Both of these water molecules are positioned close to the Asp145 carboxylate. One, located only $2.8 \AA$ away, is within H-bonding distance. The conformation of the Asp145 side chain as well as the conformations of the other active site residues are, however, not noticeably altered.

Ligand Binding Constants. The $k_{\text {on }}$ and $k_{\text {off }}$ values for the binding of 4-CBA-CoA and 4-HBA-CoA to wild-type and H90Q dehalogenases were measured using stopped-flow
Table 1: $k_{\mathrm{on}}$ and $k_{\text {off }}$ for 4-CBA-CoA and 4-HBA-CoA Binding to Wild-Type and H90Q Dehalogenase in $50 \mathrm{mM} \mathrm{K}^{+}$Hepes $(\mathrm{pH} 7.5$, $25^{\circ} \mathrm{C}$ ) and Microscopic Rate Constants (Numbered According to Kinetic Model Shown in Scheme 2) for Wild-Type and H90Q Dehalogenase Catalyzed Dehalogenation of 4-CBA-CoA in $50 \mathrm{mM}$ $\mathrm{K}^{+}$Hepes $\left(\mathrm{pH} 7.5,25^{\circ} \mathrm{C}\right)^{a}$

\begin{tabular}{lcc}
\hline & $\begin{array}{c}\text { wild-type } \\
\text { dehalogenase }\end{array}$ & $\begin{array}{c}\text { H90Q } \\
\text { dehalogenase }\end{array}$ \\
\hline 4-CBA-CoA & $7 \pm 2$ & \\
$\quad k_{\text {on }}$ & $(2.8 \pm 0.5) \times 10^{1}$ & $(3.6 \pm 0.5) \times 10^{1}$ \\
$\quad k_{\text {off }}$ & & \\
$4-$ HBA-CoA & $(3.3 \pm 0.3) \times 10^{1}$ & $(1.1 \pm 0.2) \times 10^{1}$ \\
$k_{\text {on }}$ & $(2.5 \pm 0.5) \times 10^{1}$ & $(1.2 \pm 0.5) \times 10^{1}$ \\
$\quad k_{\text {off }}$ & $7 \pm 2$ & \\
microscopic rate constants & $8 \pm 2$ \\
$k_{1}$ & $(3 \pm 1) \times 10^{1}$ & $(4.0 \pm 0.1) \times 10^{1}$ \\
$k_{-1}$ & $(2 \pm 1) \times 10^{2}$ & $(1.0 \pm 0.5) \times 10^{2}$ \\
$k_{2}$ & $(2 \pm 1) \times 10^{3}$ & $(3 \pm 1) \times 10^{3}$ \\
$k_{-2}$ & $2 \pm 1$ & $(3 \pm 1) \times 10^{-1}$ \\
$k_{3}$ & $(2.0 \pm 0.5) \times 10^{1}$ & $(1.10 \pm 0.04) \times 10^{-2}$ \\
$k_{-3}$ & $(2.5 \pm 0.5) \times 10^{-1}$ & $(6.6 \pm 0.6) \times 10^{-1}$ \\
$k_{4}$ & $1.5 \pm 0.2$ & $(5.0 \pm 0.5) \times 10^{-3}$ \\
$k_{-4}$ & $8 \pm 0.8$ & $(1.1 \pm 0.1) \times 10^{-1}$ \\
$k_{5}$ & $k_{-5}$ & \\
\hline
\end{tabular}

${ }^{a}$ The unimolecular rate constants are reported in units of $\mathrm{s}^{-1}$ while the bimolecular rate constants are reported in units of $\mathrm{s}^{-1} \mu \mathrm{M}^{-1}$. See Results for further details.

fluoresence quenching techniques. The values obtained are listed in Table 1 . We note that because the $k_{\text {off }}$ values are derived by data extrapolation (see Experimental Procedures), they are defined with less accuracy than are the $k_{\text {on }}$ values. Also, we note that the $k_{\text {off }}$ values reflect the dissociation of the ligand from enzyme that has not undergone catalysis. The significance of this distinction will be revealed in the following section.

Time Courses for Product Formation Measured Using Multiple Turnover Conditions. Wild-type and H90Q dehalogenases were reacted at high concentration with 4-CBACoA present in a 10-fold (or greater) excess. Product formation was monitored over the course of several catalytic turnovers. The plot of product concentration vs reaction time, measured for the reaction catalyzed by wild-type dehalogenase (Figure 4A), indicates that the rate of product formation for the first turnover, $2.3 \mathrm{~s}^{-1}$, is slightly faster than that, 0.7 $\mathrm{s}^{-1}$, observed for the turnovers which follow. The $k_{\text {cat }}$ for wild-type dehalogenase measured using steady-state initial velocity techniques is $0.6 \mathrm{~s}^{-1}(5)$, which is in close agreement 

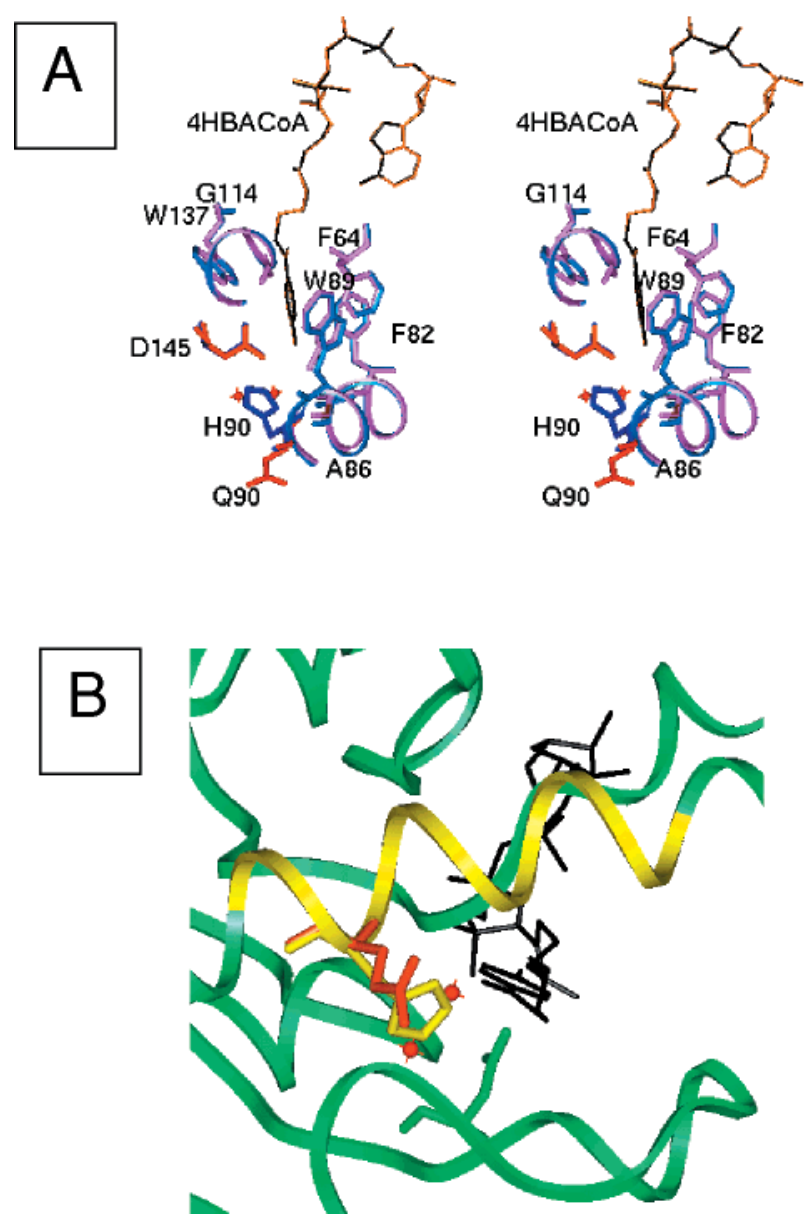

FIGURE 3: (A) Illustration of the two active sites observed in the superposition of the structures of the 4-HBA-CoA-wild-type dehalogenase complex (10) and 4-HBA-CoA-H90Q dehalogenase complex (generated using Insight II). The backbone segments and residues of the wild-type dehalogenase are pictured in pastel and royal blue, while those of the H90Q mutant are pictured in pink and red. The two red spheres represent water molecules observed in the H90Q structure only. The 4-HBA-CoA ligand bound to wildtype dehalogenase is shown in black while that bound to the H90Q dehalogenase is shown in gold. (B) Active site region of the 4-HBACoA-wild-type dehalogenase complex shown from the side opposite to the active site entrance. The $\alpha$-helix that creates the bottom wall of the active site is shown in yellow. The H90 side chain that enters into the active site is also pictured in yellow. The Asp145 is colored green and the 4-HBA-CoA ligand black. The 4-HBA-CoA-H90Q dehalogenase is superpositioned, but only the Q90 residue (red) and the two water molecules (red) are shown.

with the rate constant derived for multiple turnovers $(0.7$ $\mathrm{s}^{-1}$ ) (Figure 4A). Thus, the rate of conversion of the enzyme-substrate complex to the enzyme-product complex appears to be 3-fold faster than is the net rate of the sequence of steps leading from one enzyme-product complex to the next. This sequence includes the release of $\mathrm{Cl}^{-}, \mathrm{H}^{+}$, and 4-HBA-CoA from the $\mathrm{E} \cdot 4-\mathrm{HBA}-\mathrm{CoA} \cdot \mathrm{Cl}^{-} \cdot \mathrm{H}^{+}$complex, a possible conformational change in the enzyme preceding or following product release, the binding of substrate to the free enzyme, and, finally, catalysis.

The difference observed in single and multiple turnover rates associated with wild-type dehalogenase catalysis was noted in an earlier study $(5,22)$ wherein product formation curves were determined for the reaction of $\left[{ }^{14} \mathrm{C}\right] 4-\mathrm{CBA}-\mathrm{CoA}$ with wild-type dehalogenase present in a 1:1 vs a $12: 1$ ratio. The rate of product formation in the former reaction was 3
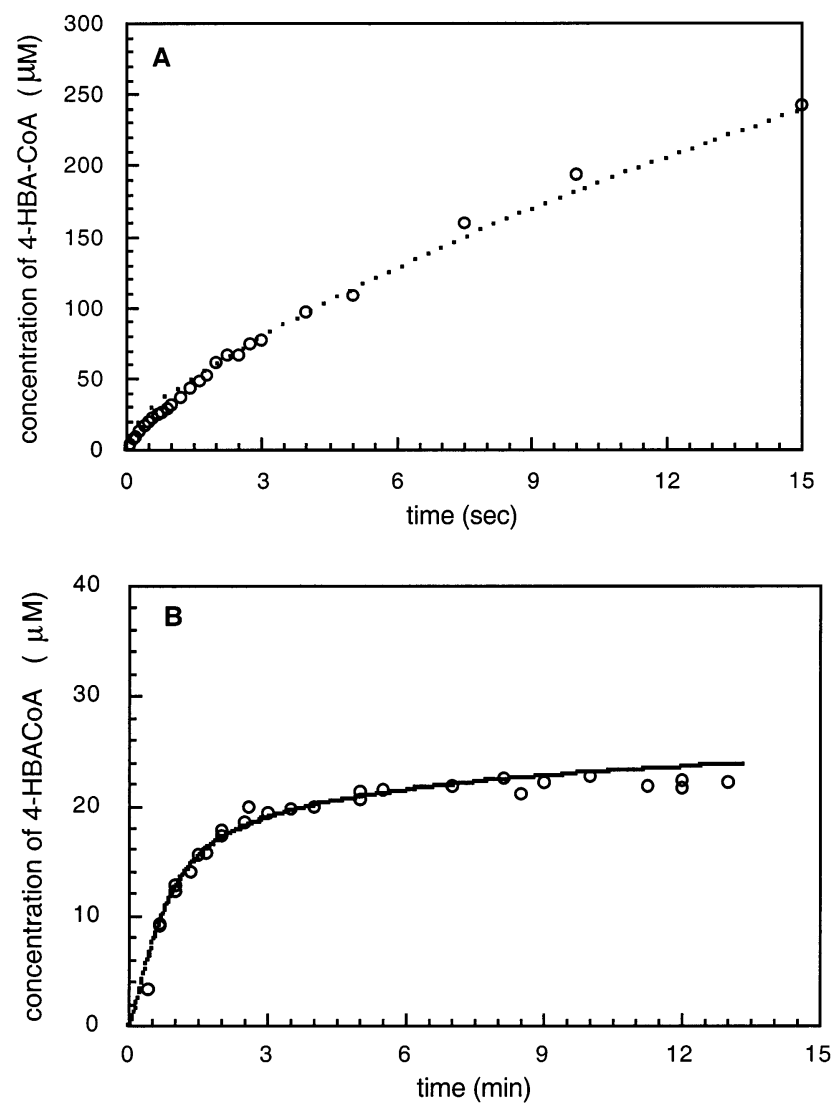

FIgURE 4: (A) Time course for 4-HBA-CoA formation in the reaction of $1500 \mu \mathrm{M} 4-\mathrm{CBA}-\mathrm{CoA}$ and $30 \mu \mathrm{M}$ wild-type dehalogenase in $50 \mathrm{mM} \mathrm{K}{ }^{+}$Hepes $\left(\mathrm{pH} \mathrm{7.5,25}{ }^{\circ} \mathrm{C}\right)$. The dashed curve was generated with KINSIM using the kinetic model shown in Scheme 2 and the following rate constants: $k_{1}=7 \mu \mathrm{M}^{-1} \mathrm{~s}^{-1}, k_{-1}$ $=28 \mathrm{~s}^{-1}, k_{2}=120 \mathrm{~s}^{-1}, k_{-2}=2000 \mathrm{~s}^{-1}, k_{3}=42 \mathrm{~s}^{-1}, k_{-3}=1 \mathrm{~s}^{-1}$, $k_{4}=25 \mathrm{~s}^{-1}, k_{-4}=0.3 \mathrm{~s}^{-1}, k_{5}=1.5 \mathrm{~s}^{-1}, k_{-5}=8 \mu \mathrm{M}^{-1} \mathrm{~s}^{-1}$. (B) Time course for 4-HBA-CoA formation in the reaction of $200 \mu \mathrm{M}$ 4-CBA-CoAand $20 \mu \mathrm{M}$ H90Q dehalogenase in $50 \mathrm{mM} \mathrm{K} \mathrm{K}^{+} \mathrm{Hepes}$ $\left(\mathrm{pH} 7.5,25^{\circ} \mathrm{C}\right)$. The dashed curve was generated with KINSIM using the kinetic model shown in Scheme 2 and the following rate constants: $k_{1}=7 \mu \mathrm{M}^{-1} \mathrm{~s}^{-1}, k_{-1}=36 \mathrm{~s}^{-1}, k_{2}=120 \mathrm{~s}^{-1}, k_{-2}=$ $2000 \mathrm{~s}^{-1}, k_{3}=0.42 \mathrm{~s}^{-1}, k_{-3}=0.01 \mathrm{~s}^{-1}, k_{4}=0.14 \mathrm{~s}^{-1}, k_{-4}=$ $0.006 \mathrm{~s}^{-1}, k_{5}=0.0005 \mathrm{~s}^{-1}, k_{-5}=0.11 \mu \mathrm{M}^{-1} \mathrm{~s}^{-1}$.

$\mathrm{s}^{-1}$, and in the latter (averaged over six turnovers), $0.6 \mathrm{~s}^{-1}$. It was speculated that this rate difference was caused by product inhibition (the $K_{\mathrm{d}}$ of 4-HBA-CoA is $2 \mu \mathrm{M}$ ), which is not possible in the single turnover reaction, but is possible in the multiple turnover reaction. In the present study, we measured the steady-state rate constant under conditions expected to minimize product inhibition (viz., $1 \%$ conversion of $1 \mathrm{mM}$ substrate) and found that it, too, is $0.6 \mathrm{~s}^{-1}$. Therefore, the comparatively slow steady-state rate is not caused by product inhibition, but rather by a rate-limiting step which takes place after catalysis has occurred.

The time course for product formation catalyzed by H90Q dehalogenase is shown in Figure 4B. Product formation was measured for up to $3 \mathrm{~h}$; however, for illustration purposes, only the data measured for the first $15 \mathrm{~min}$ are shown. The difference observed in the rates of the first turnover and the second turnover is dramatic. The rate data were fitted with eq 1, as described under Experimental Procedures, to obtain the rate constant for the first turnover, $k=1.6 \times 10^{-2} \mathrm{~s}^{-1}$, and the rate constant for subsequent turnovers, $k=2 \times 10^{-4}$ $\mathrm{s}^{-1}$. A comparison of the rate constants obtained for the first 
turnover of bound substrate catalyzed by wild-type and H90Q dehalogenases $\left(2.3\right.$ vs $\left.1.6 \times 10^{-2} \mathrm{~s}^{-1}\right)$ shows that the mutation slows down catalysis 144-fold. Moreover, the mutation has slowed the rate-limiting step following catalysis $\sim 3500$-fold (from 0.7 to $2 \times 10^{-4} \mathrm{~s}^{-1}$ ).

Time Courses for Substrate, Covalent Enzyme, and Product Measured for a Single Turnover Reaction. The next step in the comparative kinetic analysis of the wild-type and H90Q dehalogenases was to determine the rate constants for the individual reaction steps catalyzed. As pointed out in the introduction, dehalogenase catalysis may proceed via the two covalent intermediates EMc and EAr. Time courses for the consumption of substrate, formation of product, and formation and consumption of the covalent enzyme intermediates were measured for the reaction of $43 \mu \mathrm{M}\left[{ }^{14} \mathrm{C}\right] 4-$ CBA-CoA and $78 \mu \mathrm{M}$ wild-type or H90Q dehalogenase, using filtration methods to separate the enzyme from acidquenched reaction samples, and HPLC to separate the unconsumed substrate from the $\left[{ }^{14} \mathrm{C}\right] 4-\mathrm{HBA}-\mathrm{CoA}$. The covalent attachment of the radiolabel to the denatured enzyme was demonstrated by separating the quenched reaction mixture on a SDS-PAGE gel (shown in reference 23) and observing the coincidence of the ${ }^{14} \mathrm{C}$-label and the protein band.

The rate data shown in Figure 5A,B were fitted using a kinetic model that includes steps $2-4$ of the kinetic model shown in Scheme 2, and the computer program Dynafit (17). We initially assumed, and then verified by fitting analysis, ${ }^{2}$ that the isolated radiolabeled enzyme (16\% maximum accumulation for wild-type dehalogenase and $7 \%$ for the H90Q mutant) is derived from both EAr and EMc. The quenched EMc intermediate releases $\mathrm{Cl}^{-}$to form the arylated enzyme.

Transient Kinetic Data Analysis. As stated in the previous paragraph, the microscopic rate constants associated with the forward and reverse directions of EMc $\left(k_{2} / k_{-2}\right)$, $\operatorname{EAr}\left(k_{3} / k_{-3}\right)$, and $\mathrm{E} \cdot 4-\mathrm{HBA}-\mathrm{CoA} \cdot \mathrm{H}^{+} \cdot \mathrm{Cl}^{-}\left(k_{4} / k_{-4}\right)$ formation were first obtained by using the Dynafit computer program (17) to analyze the single turnover rate data plotted in Figure 5A,B. The progress curves generated by this fitting procedure are shown in Figure 5A,B. The absence of separate progress curves for EMc and EAr limits the certainty by which the values of the rate constants associated with EMc and EAr formation can be defined. To explore the boundaries of these rate constants, the computer program KINSIM (18) was employed to simulate progress curves for a large range of rate constant values. The values of the microscopic rate

2 The kinetic model used in data fitting, and in curve simulation, holds that two covalent intermediates, EMc and EAr, are formed in the reaction. Data fitting to a simplified model in which the EMc and EAr steps are combined (EMc thus represents a transition state rather than an intermediate) was also tried. Both kinetic models provide good fits to the rate data obtained for wild-type dehalogenase, but only the two-covalent intermediate model gives a good fit to the H90Q dehalogenase rate data. We believe that this is because the rates of the two steps are better resolved in the H90Q-catalyzed reaction. Independent evidence for the intermediacy of EMc derives from a stoppedflow absorption kinetic analysis of the dehalogenase-catalyzed reactions of 4-CBA-CoA analogues (12). These findings, coupled with "chemical logic" (there is ample evidence in the literature for stepwise nucleophilic substitution on activated aromatic rings but none supporting a concerted mechanism) (24-27), are most consistent with the two-covalent intermediate kinetic model of Scheme 2, and thus support its use in the determination of the microscopic rate constants.
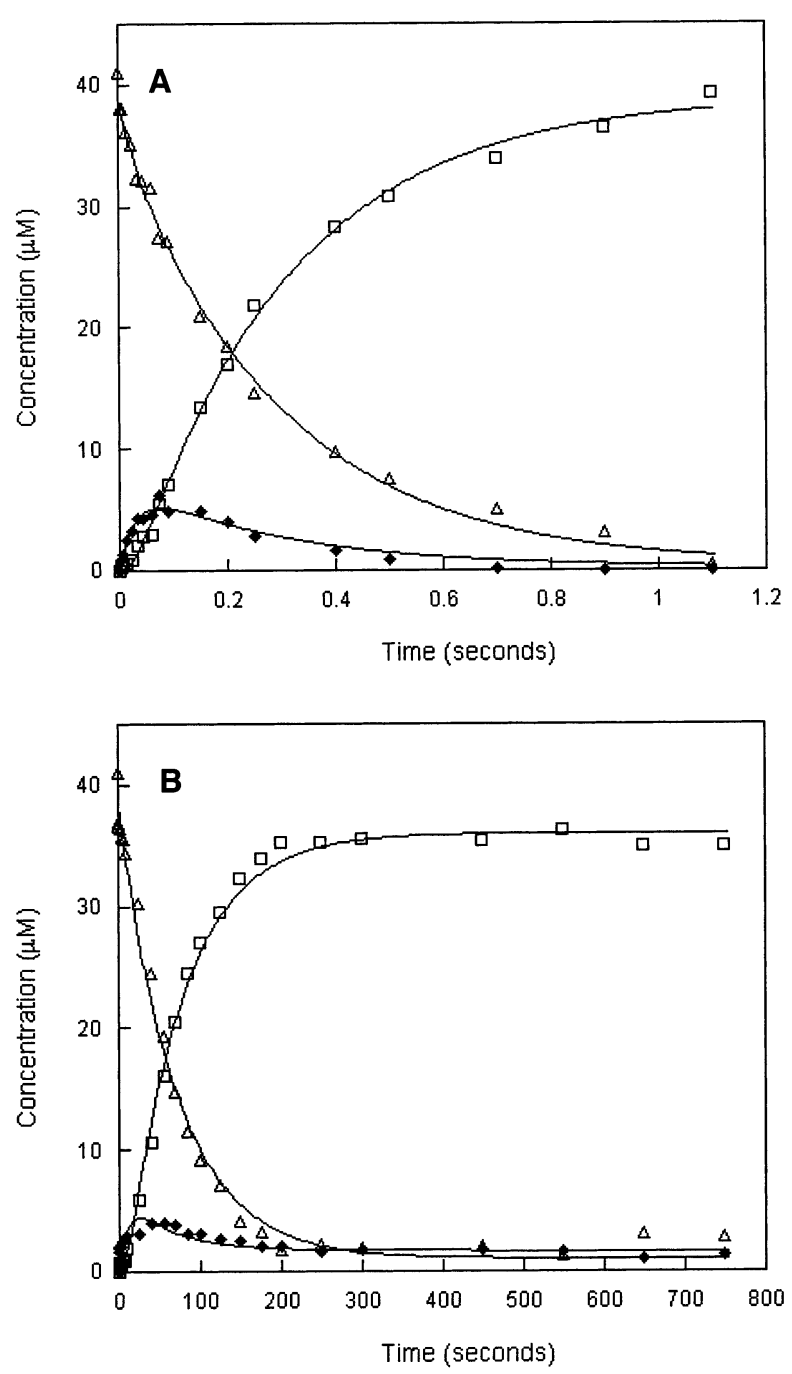

FIGURE 5: Time courses for a single turnover reaction of (A) wildtype and (B) H90Q dehalogenases $(78 \mu \mathrm{M})$ with $\left[{ }^{14} \mathrm{C}\right] 4-\mathrm{CBA}-\mathrm{CoA}$ $(43 \mu \mathrm{M})$ in $50 \mathrm{mM} \mathrm{K}{ }^{+}$Hepes $\left(\mathrm{pH} 7.5,25^{\circ} \mathrm{C}\right)$ measured by rapid quench. $(\triangle)\left[{ }^{14} \mathrm{C}\right] 4-\mathrm{CBA}-\mathrm{CoA},(\square)\left[{ }^{14} \mathrm{C}\right] 4-\mathrm{HBA}-\mathrm{CoA},(\diamond){ }^{14} \mathrm{C}-$ labeled enzyme. The curves were generated using the computer program Dynafit (17).

constants used as "in put" for the simulations were varied (singularly or in pairs) from the values determined by the Dynafit-based analysis. The simulated curves generated from the varied rate constant(s) were evaluated by visual inspection for closeness of fit to the experimental data. The range of a rate constant value that provided a "good fit" to the data was used to define the boundary of that rate constant. These boundaries are listed as the degrees of uncertainties $( \pm)$ in each of the rate constants defined by the Dynafit-based analysis (Table 1).

The steps involving substrate binding and product release were initially examined using stopped-flow fluorescence quenching techniques as described in the previous section. This approach adequately defined the substrate binding constants $k_{1}$ and $k_{-1}$. On the other hand, the $k_{\text {on }}$ and $k_{\text {off }}$ for 4-HBA-CoA binding to the free enzyme may be significantly different from the rate constants governing 4-HBA-CoA binding to $\mathrm{E} \cdot \mathrm{H}^{+} \cdot \mathrm{Cl}^{-}$and dissociating from $\mathrm{E} \cdot 4-\mathrm{HBA}-\mathrm{CoA} \cdot$ $\mathrm{H}^{+} \cdot \mathrm{Cl}^{-}$. (It is likely that $4-\mathrm{HBA}-\mathrm{CoA}$ is released first from the enzyme, since the $\mathrm{Cl}^{-}$and $\mathrm{H}^{+}$products are bound by residues located at the bottom of the active site, below the 4-HBA-CoA ligand.) In the case of the H90Q mutant, we 
have been able to measure by stopped-flow absorption techniques (data not shown) the relative rates of 4-HBACoA release from the $\mathrm{E} \cdot 4-\mathrm{HBA}-\mathrm{CoA}$ complex (formed by mixing enzyme with 4-HBA-CoA) vs from the E-4-HBA$\mathrm{CoA} \cdot \mathrm{H}^{+} \cdot \mathrm{Cl}^{-}$complex (formed by mixing enzyme with substrate and allowing time for a catalytic turnover). The former is 3-fold faster than the latter. Thus, at least in the case of the H90Q mutant, the reported $k_{\text {off }}$ exceeds the actual rate constant by a factor of 3 .

Once 4-HBA-CoA dissociates from the E-4-HBA-CoA $\mathrm{H}^{+} \cdot \mathrm{Cl}^{-}$complex, the $\mathrm{Cl}^{-}$and $\mathrm{H}^{+}$products must then dissociate, and the enzyme may have to undergo a conformational change and/or proton reshuffling step (see, for example, reference 28). At present, we do not know the values of the rate constants associated with these steps. For this reason, these rate constants, along with that governing the dissociation of 4-HBA-CoA, have been group into the single rate constant $k_{5}$ (see Scheme 2). Likewise, the rate constants governing the reverse steps are grouped into the single rate constant $k_{-5}$. Based on the rate data shown in Figure 4A,B, we know that for the wild-type enzyme, the culmination of the rates of these steps is partially ratelimiting. For the H90Q dehalogenase, the culmination of the rates of these steps is remarkably slow.

Using KINSIM along with the microscopic rate constants for the chemical steps $\left(k_{2} / k_{-2}, k_{3} / k_{-3}\right.$, and $\left.k_{4} / k_{-4}\right)$ to simulate curves to the rate data shown in Figure 4A,B, the values of the forward and reverse microscopic rate constants $k_{5}$ and $k_{-5}$ (Table 1) were defined. For wild-type dehalogenase, $k_{-5}$ $=8 \mu \mathrm{M}^{-1} \mathrm{~s}^{-1}$ and $k_{5}=1.5 \mathrm{~s}^{-1}$. The 7-fold difference between $k_{\text {off }}$ and $k_{5}$ may reflect slower release of 4-HBA$\mathrm{CoA}$ from the $\mathrm{E} \cdot 4-\mathrm{HBA}-\mathrm{CoA} \cdot \mathrm{H}^{+} \cdot \mathrm{Cl}^{-}$complex than from the $\mathrm{E} \cdot 4-\mathrm{HBA}-\mathrm{CoA}$ complex, or a partially rate-limiting $\mathrm{H}^{+}$ or $\mathrm{Cl}^{-}$release, or a slow conformational change and/or proton shuffling step in route to the catalytically active form of the enzyme. For H90Q, $k_{-5}=0.1 \mu \mathrm{M}^{-1} \mathrm{~s}^{-1}$ and $k_{5}=0.00005$ $\mathrm{s}^{-1}$. Since the release of 4-HBA-CoA from the E-4-HBA$\mathrm{CoA} \cdot \mathrm{H}^{+} \cdot \mathrm{Cl}^{-}$complex is only 3-fold slower than it is from the E-4-HBA-CoA complex $\left(k_{\text {off }}=12 \mathrm{~s}^{-1}\right)$, it appears that the replacement of His 90 with Gln has strongly inhibited $\mathrm{H}^{+}$or $\mathrm{Cl}^{-}$release, or a conformational change and/or proton shuffling step in route to the catalytically active form of the enzyme.

Using the 12 microscopic rate constants, within their defined limits, the theoretical $k_{\text {cat }}$ and $K_{\mathrm{m}}$ values for wildtype dehalogenase were calculated. For wild-type dehalogenase, the calculated values are $k_{\text {cat }}=0.8 \mathrm{~s}^{-1}$ and $K_{\mathrm{m}}=$ $3.7 \mu \mathrm{M}$. These values compare well with the experimental values of $k_{\text {cat }}=0.6 \mathrm{~s}^{-1}$ and $K_{\mathrm{m}}=3.7 \pm 0.4 \mu \mathrm{M}$ (5). We were not able to measure the steady-state $k_{\text {cat }}$ and $K_{\mathrm{m}}$ values for the H90Q mutant because slow turnover, coupled with tight substrate binding, made it technically impossible to adhere to the conditions required for steady-state kinetics (viz., $[\mathrm{S}] \gg[\mathrm{E}]$ at $[\mathrm{S}] \geq K_{\mathrm{m}}$ ). Thus, a comparison between experimental and theoretical values cannot be made at this time.

\section{DISCUSSION}

Comparison of the Microscopic Rate Constants Governing Wild-Type and H90Q Dehalogenase Catalysis. The rate of an enzyme-catalyzed reaction is determined by the micro- scopic rate constants governing the substrate binding step(s), each chemical step, and the product release step(s). A rate-limiting isomerization step, involving an enzyme conformational change or proton exchange, may also contribute. A mutation made to the active site can change any one of these microscopic rate constants and, hence, the overall rate of catalysis. In determining the contribution made by a particular active site residue in catalysis using amino acid replacement, it is advantageous to measure the impact of the mutation on each microscopic rate constant. As demonstrated here, with the H90Q dehalogenase, an amino acid replacement made to inhibit one particular step can inhibit other steps as well.

The original goal of our study was to obtain evidence for the role of the active site residue His 90 in the hydrolysis of the EAr intermediate formed during dehalogenase-catalyzed dehalogenation of 4-CBA-CoA to 4-HBA-CoA. The His90 was replaced with Gln, and the structures and catalytic properties of the wild-type and mutant enzymes were compared. The product formation curves (illustrated in Figure 4A,B) showed that a step following product formation on the enzyme is partially rate-limiting in catalysis by the wildtype enzyme and completely rate-limiting in catalysis by the H90Q mutant. The three chemical steps (Figure 1A) are catalyzed by the wild-type enzyme 144-fold faster than they are catalyzed by the mutant. Within the resolution of the transient kinetic analysis, the rate of the first step of the reaction ( $\mathrm{ES} \rightarrow \mathrm{EMc}$ ) is not noticeably changed in the mutant. On the other hand, the rate of the EMc $\rightarrow \mathrm{E} \cdot \mathrm{Ar}^{\cdot} \mathrm{Cl}^{-}$ step is decreased 133-fold, and the rate of the $\mathrm{EAr}^{\cdot} \mathrm{Cl}^{-} \rightarrow$ $\mathrm{E} \cdot \mathrm{P} \cdot \mathrm{H}^{+} \cdot \mathrm{Cl}^{-}$step is decreased 154 -fold.

The microscopic rate constants obtained for the first four steps of dehalogenase catalysis were used to extract the rate constant governing the steps associated with enzyme recycling following product formation. For wild-type dehalogenase, the value of this rate constant is $1.5 \mathrm{~s}^{-1}$, which proves to be partially rate-limiting. Otherwise, the rate constants for the forward directions of the reaction steps are reasonably well balanced. The bottleneck in catalysis by the wild-type dehalogenase is the EMc-forming step, for which the rate constant for the reverse direction is 10 -fold larger than that for the forward direction.

For the H90Q mutant, the value obtained for the "recycling" rate constant is $2 \times 10^{-4} \mathrm{~s}^{-1}$. The steps following product formation thus severely limit the overall rate of catalysis. Despite the difficulties imposed on the chemical steps resulting from the substitution of the His 90 with Gln, the most difficult step in H90Q dehalogenase catalysis appears to be release of the proton and/or chloride ion from the active site or, more likely, a conformational change and/ or proton shuffling step which resets the active site for catalysis.

Analysis of the Kinetic Changes in the H90Q Dehalogenase within the Context of the Active Site Structural Changes. To gain insight into why Gln substitution of H90 has an inhibitory effect on both EAr formation and hydrolysis, a comparison of the two active sites was made (Figure 3A,B). The positions of the catalytic residues are not noticeably changed in the H90Q mutant. The one exception is the site of substitution. Whereas the His90 imidazole side chain projects into the active site, the Gln90 side chain is rotated away from it. The movement of the side chain out of the 
active site creates a small hole at the bottom of the active which allows solvent to access the catalytic residues. Hydration of the active site, as observed in the H90Q dehalogenase structure (Figure 3B), is likely to have a significant impact on EAr formation.

EAr formation requires dissociation of chloride ion from the benzoyl ring of the Meisenheimer complex. As pictured in Figure 1B, the aromatic rings of Phe64, Phe82, and Trp89 form a "receiving pocket" for the displaced chloride ion (the His90 is located too far away to assist). Electrostatic interactions between these aromatic residues and the anion are highly dependent on the dielectric constant of the surrounding medium (29-31). Inclusion of water molecules at this site will no doubt cause a significant change in the local environment. Such alteration may underlie the 133 -fold reduction observed in the rate constant for EAr formation.

EAr hydrolysis involves the displacement of the phenolate anion from the Asp145 $\mathrm{C}=\mathrm{O}$ by an activated water molecule. The presumed role of His 90 is activation of this attacking water. As seen from the X-ray structure of the wild-type dehalogenase-4-HBA-CoA complex, the His90 N(1)H is engaged in a $\mathrm{H}$-bond interaction with the backbone carbonyl of Ala86 (Figure 3A). This interaction may serve to direct the $\mathrm{N}(3)$ of the imidazole ring toward the carboxylate group of Asp145, located $3.6 \AA$ away. One might assume that a water molecule is held between the His 90 and the $\mathrm{C}=\mathrm{O}$ of Asp145 in EAr, through H-bond interaction with the His90 imidazole N(3). In the absence of the imidazole side chain, this water molecule cannot be oriented or activated for nucleophilic attack. Loss of general base catalysis by His 90 would thus contribute to the observed 154-fold reduction in rate of EAr hydrolysis in the H90Q mutant.

Summary of the Roles of His90 in Dehalogenase Catalysis. First, from the X-ray structural data, it is seen that His 90 serves as barrier between solvent and the active site (Figure 3B). The His90 side chain is held in the active site through its interaction with the backbone amide $\mathrm{C}=\mathrm{O}$ of Ala86. Substitution of His90 disrupts this interaction and, hence, the forces that secure the barrier to solvent. Hydration of the active site perturbs the electrostatic environment which, in turn, impairs the dissociation of $\mathrm{Cl}^{-}$ion from the EMc intermediate.

Second, His90 functions in general base catalysis of the hydrolysis of the EAr intermediate. The rate contribution made by the His90 in EAr hydrolysis is modest in comparison to the catalytic contribution of the His functioning in haloalkane dehalogenase (32). Like the 4-CBA-CoA dehalogenase, the haloalkane dehalogenase employs an active site Asp in nucleophilic catalysis and a His residue in general base catalysis $(32,33)$. However, the loss of the catalytic His function in the haloalkane dehalogenase halts the progress of the reaction at the ester intermediate (33), while loss of the His 90 function in the 4-CBA-CoA dehalogenase merely slows down the rate of ester hydrolysis 154 -fold. The high energy of the alkoxide ion leaving group generated from the alkylated haloalkane dehalogenase necessitates a twostep mechanism with His fulfilling the dual roles of base and acid catalyst. This may account for why the active site His is essential to catalytic turnover. In the case of 4-CBACoA dehalogenase EAr hydrolysis, the "low energy" benzoyloxide ion leaving group [solution $\mathrm{p} K_{\mathrm{a}}$ of the 4-HBA$\mathrm{CoA} \mathrm{C}(4) \mathrm{OH}$ is 8.6] (34) does not necessitate protonation.
Indeed, based on the recent work of Henge and Cleland (35, 36) on ester hydrolysis mechanisms, one might predict that the hydrolysis occurs by a concerted mechanism. Accordingly, His90 functions solely as a general base, consistent with the 154-fold reduction in the rate of EAr hydrolysis observed in its absence (37).

Finally, His90 is important to the regeneration of active enzyme following the turnover of substrate to product in the active site. The culmination of steps involved in this process occurs 3500-fold slower in the H90Q mutant compared to the wild-type enzyme. In future studies, we aim to identify these steps and the role that His 90 may play in their execution.

\section{ACKNOWLEDGMENT}

The assistance of Mr. Zhihao Zhuang and Ms. Dongmei Ye in preparing Figures 2 and 3 is gratefully acknowledged. We also thank Dr. Peter Kuzmic for making the Dynafit computer program available for use.

\section{NOTE ADDED AFTER PRINT PUBLICATION}

Because of an error, the Protein Data Bank entry for the X-ray coordinates appeared as $1 \mathrm{JX} 2$ instead of as $1 \mathrm{JXZ}$ in the version published on the Web 10/19/01 (ASAP) and in the November 13, 2001, issue (Vol. 40, No. 45, pp 1347413482); the correct electronic version of the paper was published 12/05/02, and an Addition and Correction appears in the December 31, 2002, issue (Vol. 41, No. 52).

\section{REFERENCES}

1. Dunaway-Mariano, D., and Babbitt, P. C. (1994) Biodegradation 5, 259-276.

2. Fetzner, S. (1998) Appl. Microbiol. Biotechnol. 50, 633-657.

3. Ornston, L. N. (1990) in Proceedings of the Symposium on Genetics of Industrial Organisms (Heslot, H., Davies, J., Bibichon, L., Duran, G., and Penasse, L., Eds) Vol. 2, p 1061, Microbiologie: Societe Francaise de Microbiologie, Strasbourg.

4. Crooks, G. P., Xu, L., Barkley, R. M., and Copley, S. D. (1995) J. Am. Chem. Soc. 117, 10791-10798.

5. Liang, P. H., Yang, G., and Dunaway-Mariano, D. (1993) Biochemistry 32, 12245-12250.

6. Taylor, K. L., Liu, R. Q., Liang, P. H., Price, J., Dunaway-Mariano, D., Tonge, P. J., Clarkson, J., and Carey, P. R. (1995) Biochemistry 34, 13881-13888.

7. Yang, G., Liang, P. H., and Dunaway-Mariano, D (1994) Biochemistry 33, 8527-8531.

8. Taylor, K. L., Xiang, H., Liu, R. Q., Yang, G., and DunawayMariano, D. (1997) Biochemistry 36, 1349-1361.

9. Clarkson, J., Tonge, P. J., Taylor, K. L., Dunaway-Mariano D., and Carey, P. R. (1997) Biochemistry 36, 10192-10199.

10. Benning, M. M., Taylor, K. T., Liu, R. Q., Yang, G., Xiang, H., Wesenberg, G., Dunaway-Mariano, D., and Holden, H. M. (1996) Biochemistry 35, 8103-8109.

11. Dong, J., Xiang, H., Luo, L., Dunaway-Mariano, D., and Carey, P. R. (1999) Biochemistry 38, 4198-4206.

12. Liu, R. Q., Liang, P. H., Scholten, J., and Dunaway-Mariano, D. (1995) J. Am. Chem. Soc. 117, 5003-5004.

13. Yang, G., Liu, R. Q., Taylor, K. L., Xiang, H., Price, J., and Dunaway-Mariano, D. (1996) Biochemistry 35, 10879-10885.

14. Chang, K. H., Liang, P. H., Beck, W., Scholten, J. D., and Dunaway-Mariano, D. (1992) Biochemistry 31, 5605-5610.

15. Bradford, M. (1976) Anal. Biochem. 72, 248-254.

16. Johnson, K. A. (1992) The Enzymes, pp 36-37, Academic Press, New York.

17. Kuzmic, P. (1996) Anal. Biochem. 237, 260-273.

18. Barshop, B. A., Wrenn, R. F., and Frieden, C. (1983) Anal. Biochem. 130, 134-145.

19. Segal, H. I. (1993) Enzyme Kinetics, pp 505-845, John Wiley \& Sons, Inc., New York. 
20. Kabsch, W. (1988) J. Appl. Crystallogr. 21, 67-71.

21. Kabsch, W. (1988) J. Appl. Crystallogr. 21, 916-924.

22. Liang, P. H. (1994) Ph.D. Thesis, pp 107-112, University of Maryland, College Park, MD.

23. Yang, G. (1995) Ph.D. Thesis, pp 59-62, University of Maryland, College Park, MD.

24. Bernasconi, C. F. (1980) Chimica 31, 1.

25. Chen, W. J., Griminski, G. F., and Armstrong, R. N. (1988) Biochemistry 27, 647-654.

26. Griminski, G. F., Zhang, P., Sesay, M., Ammon, H., and Armstrong, R. N. (1989) Biochemistry 28, 6252-6258.

27. Morgenstern, R., Svensson, R., Bernat, B. A., and Armstrong, R. N. (2001) Biochemistry 40, 3378-3384.

28. Rose, I. A. (1998) Biochemistry 37, 17651-17658.

29. Thomas, K. A., Smith, G. M., Thomas, T. B., and Feldman, R. J. (1982) Proc. Natl. Acad. Sci. U.S.A. 79, 4843-4847.

30. Burley, S. K., and Petsko, G. A. (1986) FEBS Lett. 203, 139143.
31. Warshel, A. (1998) J. Biol. Chem. 273, 27035-27038.

32. Pries, F., Kingman, J., Krooshof, G. H., Jeronimus-Stratingh, Bruins, A. P., and Janssen, D. B. (1995) J. Biol. Chem. 270 , 10405-10411

33. Pries, F., Kingman, J., Pentenga, M., van Pouderoyen, G., Jeronimus-Stratingh, Bruins, A. P., and Janssen, D. B. (1994) Biochemistry 33, 1242-1247.

34. Webster, L. T., Mieyel, J. J., and Siddiqui, U. A. (1974) J. Biol. Chem. 249, 2641-2645.

35. Hengge, A. C., and Hess, R. A. (1994) J. Am. Chem. Soc. 116, $11256-11263$

36. Hess, R. A., Hengge, A. C., and Cleland, W. W. (1998) J. Am. Chem. Soc. 120, 2703-2709.

37. Fersht, A. (2000) Structure and Mechanism in Protein Science, 3rd ed., p 66, W. H. Freeman \& Co., New York.

BI0114426 